PALEO

Revue d'archéologie préhistorique

25 | 2014

Varia

\title{
Intérêts de l'utilisation des décapages lors des fouilles archéologiques pour l'étude des restes de petits vertébrés
}

The advantage of using independent subdivisions during archaeological excavations for the study of small vertebrate remains

\section{Aurélien Royer}

\section{OpenEdition \\ Journals}

Édition électronique

URL : http://journals.openedition.org/paleo/2819

DOI : $10.4000 /$ paleo.2819

ISSN : 2101-0420

Éditeur

SAMRA

Édition imprimée

Date de publication : 28 décembre 2014

Pagination : $277-286$

ISSN : $1145-3370$

Référence électronique

Aurélien Royer, «Intérêts de l'utilisation des décapages lors des fouilles archéologiques pour l'étude des restes de petits vertébrés », PALEO [En ligne], 25 | 2014, mis en ligne le 28 juillet 2015, consulté le 10 décembre 2020. URL : http://journals.openedition.org/paleo/2819; DOI : https://doi.org/10.4000/ paleo.2819

\section{(9) $(\Theta \Theta$}

PALEO est mis à disposition selon les termes de la licence Creative Commons Attribution - Pas d'Utilisation Commerciale - Pas de Modification 4.0 International. 


\title{
Intérêts de l'utilisation des décapages lors des fouilles archéologiques pour l'étude des restes de petits vertébrés
}

\author{
Aurélien ROYER ${ }^{(1)}$
}

\begin{abstract}
Résumé : Les restes de petits vertébrés de nombreuses collections sont conditionnés et étudiés selon les couches archéologiques caractérisées lors de la fouille. De facto, l'analyse de ces collections est influencée par ces ensembles prédéfinis, ce qui biaise d'éventuelles réévaluations et de nouveaux découpages biostratigraphiques. S'appuyant sur l'analyse de trois collections de petits vertébrés issues de gisements fouillés à différentes périodes (les sites d'Olha I dans les Pyrénées-Atlantiques, des Pradelles en Charente et de Peyrazet dans le Lot), ce travail met en évidence l'avantage d'enregistrer précisément la provenance spatiale de ce type de microvestiges. Cet article propose donc que les restes de petits vertébrés soient systématiquement enregistrés et collectés par décapages successifs (ou passes) dont la situation spatiale est définie non pas par rapport à une couche archéologique, mais par des coordonnées cartésiennes. Ce mode d'enregistrement permet d'établir des biostratigraphies réellement indépendantes des autres stratigraphies (i.e. litho-, archéo-, chronostratigraphies) et corrélables à ces dernières.
\end{abstract}

Mots-clés : biostratigraphie, petits vertébrés, mode d'enregistrement des données, décapages.

Abstract: The advantage of using independent subdivisions during archaeological excavations for the study of small vertebrate remains. The remains of small vertebrates are generally recorded and analyzed according to archaeological levels defined during excavations. As a consequence, the study of such assemblages is influenced by these predefined levels, creating possible analytical biases and errors in the characterization of biostratigraphical units. The study of small vertebrate assemblages from Olha 1 (Pyrénées-Atlantiques), Les Pradelles (Charente) and Peyrazet (Lot) demonstrates the benefit of systematically recording detailed spatial information for these types of remains. Independent subdivisions (spits) defined by Cartesian coordinates allow independently defined biostratigraphical units to be correlated with litho- and archaeo-stratigraphy.

Key-words: biostratigraphy, small vertebrates, recording methods, spits.

\section{1 - Introduction}

Les préhistoriens sont régulièrement conduits à réévaluer les séries issues de fouilles anciennes de sites aujourd'hui considérés comme des références. Ces réévaluations sont généralement entreprises lorsqu'un « décalage » est observé entre les anciennes interprétations et les connaissances actuelles, lesquelles évoluent continuellement par suite de nouvelles découvertes conduisant à la formulation de nouvelles problématiques, de l'utilisation de nouvelles méthodes, ou bien encore par suite de la mise en place de nouveaux paradigmes analytiques. L'objectif principal des reprises de collections est donc de réviser et réactualiser les interprétations archéologiques et stratigraphiques du site sur la base du matériel issu des anciennes fouilles.

L'histoire d'un site préhistorique est une reconstitution subjective basée sur l'interprétation du contexte 
sédimentaire et géomorphologique ainsi que sur les différents vestiges archéologiques et paléontologiques exhumés. Parmi ces vestiges, les restes fauniques permettent notamment d'appréhender l'exploitation du milieu animal par l'Homme, d'établir des biostratigraphies et de reconstituer les environnements du passé. Les restes de petits vertébrés présentent pour leur part deux particularités : 1) ils sont régulièrement présents dans les gisements qui possèdent les conditions nécessaires à la fossilisation des os, et 2) ils peuvent se trouver indépendamment des vestiges des occupations humaines ou en association avec ces derniers. Néanmoins lorsqu'ils sont associés à des artéfacts, leurs accumulations résultent rarement d'activités anthropiques (Chaline 1977). Ces assemblages ne sont donc qu'exceptionnellement biaisés par l'activité de prédation des hommes, contrairement à la grande faune. En revanche, ils peuvent être influencés par le(s) agent(s) responsable(s) de leurs accumulations (e.g. Mayhew 1977 ; Andrews 1990 ; Stahl 1996). Ces petits vertébrés, principalement représentés par des rongeurs (e.g. Chaline 1977), possèdent en outre la particularité d'occuper de nombreux espaces écologiques différents. Grâce à leur petite taille, à leur grande diversité et à leur comportement plutôt sédentaire, ils reflètent un environnement local. Compte tenu de la rapidité de leur réponse aux variations du milieu, les petits vertébrés sont des témoins privilégiés des changements environnementaux survenus à proximité des gisements (e.g. Le Louarn et Quéré 2003 ; Hernández Fernández 2006 ; Auffray, Renaud, Claude 2009). De telles reconstitutions sont réalisées depuis le début du $X X^{\ominus}$ siècle (e.g. Harlé 1906 ; Bouchud 1952 ; Simard 1966 ; Chaline 1972 ; Jeannet 1978 ; Marquet 1993 ; Cuenca-Bescós et al. 2009 ; Jeannet 2010 ; López-García et al. 2010 ; Royer et al. 2013). Dans la plupart des cas, elles se réfèrent aux couches archéologiques définies lors des fouilles.

Dans cet article, deux questions sont abordées : 1) quelle est la conséquence d'un enregistrement par couches des données relatives aux petits vertébrés pour les interprétations et pour les réévaluations futures ? 2) Les subdivisions archéostratigraphiques sont-elles suffisamment pertinentes pour l'étude des paléo-associations de petits vertébrés ? Grâce à l'analyse de trois collections de petits mammifères issues de gisements fouillés à différentes périodes (les sites d'Olha I dans les Pyrénées-Atlantiques, des Pradelles en Charente et de Peyrazet dans le Lot), ce travail montre qu'il est préférable d'enregistrer et de collecter les restes de petits vertébrés par décapages successifs (ou passes). Cette méthode simple à mettre en œuvre permet de conserver des informations neutres et indépendantes qui peuvent être confrontées directement avec celles des autres vestiges archéologiques.

\section{2 - Limites et implications des couches archéologiques}

Une couche archéologique se définit comme une unité de sol constituée des dépôts laissés par les hommes ou contenant les vestiges de leurs activités (Leroi-Gourhan 1988). Elle est donc déterminée à partir de l'ensemble des différents vestiges trouvés dans le site. La couche archéologique est indépendante de la couche géologique qui se définit comme «une unité sédimentaire s'étant accumulée dans des conditions physiques constantes» (Reineck et Singh 1975, p. 82). Cette définition d'une couche archéologique est théorique. Dans la pratique, leur délimitation est délicate et relève déjà d'une interprétation du site et du matériel qui a servi à identifier ces couches (Bordes, Rigaud, Sonneville-Bordes (de) 1972). Par ailleurs, les couches archéologiques sont régulièrement définies uniquement à partir d'un premier constat de terrain qui s'appuie non pas sur l'archéologie, mais sur des critères géologiques (i.e caractéristiques des dépôts qui contiennent les artefacts tels que la texture ou la couleur). La définition des couches archéologiques est donc souvent sujette à caution et leur réalité peut être remise en cause. En conséquence, l'interprétation des vestiges pour lesquels la seule information spatiale enregistrée est la couche archéologique dont ils sont issus, est délicate (Villa 2004).

À partir des années 1930, Méroc a développé un système de cotation des objets par repères cartésiens orthonormés (Laplace-Jauretche et Méroc 1954) qui a été très rapidement employé dès le milieu du $X X^{e}$ siècle (e.g. LeroiGourhan 1950 ; Bordes et Bourgon 1951 ; SonnevilleBordes (de) et Mortureux 1955 ; Vaufrey 1956). Ce système permet d'enregistrer la position précise des vestiges au sein du gisement et présente donc l'avantage de fournir des informations neutres. II est devenu essentiel dans les méthodes de fouilles actuelles et offre la possibilité de continuer l'analyse des ensembles archéologiques après la fin des opérations de fouilles. Les collections provenant des anciennes fouilles ayant utilisé ce système peuvent ainsi être réévaluées. Néanmoins, ce système n'a été mis en œuvre que pour les objets les plus gands, identifiables lors de la fouille.

Le tamisage a été pratiqué dès la fin du $\mathrm{XIX}^{\ominus}$ siècle pour récupérer les objets qui auraient échappé à la fouille. Cependant ce n'est qu'à partir de la seconde moitié du $X X^{e}$ siècle que le tamisage devient systématique et qu'il est relié avec le carroyage du site (e.g. Lumley (de) 1969, 1972). Laplace-Jauretche (1971), dans la volonté de recueillir un maximum d'informations, propose de diviser l'ensemble du gisement en tranches de sédiments de 5 ou $10 \mathrm{~cm}$ d'épaisseur qu'il appelle respectivement demi-taille et taille. Selon lui, ces subdivisions sont «subordonnées à la stratigraphie définie par un ensemble de caractères géologiques et culturels» (1971, p.228). Néanmoins une telle définition est contraire aux règles de la stratigraphie (Hedberg 1979). Ces subdivisions ne doivent pas dépendre des séquences litho- et archéo-stratigraphiques pour être indépendantes et corrélables à ces dernières. Aujourd'hui, elles sont dénommées « décapages » ou « passes » selon la définition du dictionnaire de Préhistoire (Leroi-Gourhan 1988). Elles représentent le volume d'un hexaèdre ayant une surface de $1 / 9,1 / 4$ ou 1 mètre carré et une épaisseur variant entre 1 et $10 \mathrm{~cm}$, selon les protocoles de fouilles utilisés. Ce mode d'enregistrement permet donc, pour les pièces recueillies directement à la fouille, ainsi que pour celles obtenues par le tamisage et par le tri, de posséder 
l'ensemble des informations permettant de les replacer au sein du gisement selon des repères cartésiens.

\section{3 - Quelles interprétations peut-on obtenir d'une ancienne collection de petits vertébrés?}

Prenons l'exemple de la collection de petits vertébrés d'Olha I (Pyrénées-Atlantiques). Passemard publie en 1936 les résultats de ses fouilles du gisement d'Olha 1, qu'il réalisa entre 1916 et 1919. II décrit une séquence de près de 7 mètres de haut organisée en six ensembles stratigraphiques (Passemard 1936). L'étude des restes de petites faunes montre la présence de quatre espèces de rongeurs et deux d'insectivores. Ceux-ci proviennent d'une unique couche située en position intermédiaire entre les couches FS et FM. La révision de cette collection a montré que tous les restes osseux (mandibules, humérus, fémurs) sont entiers, ce qui suggère une collecte sélective du matériel. Cette observation conduit donc à s'interroger sur les conséquences d'une telle collecte et sur la représentativité du matériel. Par ailleurs trois espèces de rongeurs supplémentaires ont été identifiées (tabl. 1). Les aires de distributions actuelles de ces espèces se chevauchant exceptionnellement, il est légitime de se demander si leur association est «réelle» ou si elle relève d'un artefact de fouille lié à la collecte du matériel.
Finalement, la révision d'une telle série permet 1) de préciser l'identification des restes et 2 ) de reconsidérer l'intégrité de la série et de mettre en évidence de possibles biais taphonomiques. Cependant, l'absence d'information sur la localisation des restes, indépendants de leur couche d'appartenance, n'autorise pas de réinterprétations environnementales ou biostratigraphiques.

\section{4 - Avec ou sans décapages : quelles différences?}

Considérons maintenant l'exemple de Peyrazet (Lot, France). Ce gisement a livré une séquence datée du Tardiglaciaire (Langlais et al. 2009). Le protocole utilisé durant la fouille permet d'extraire les données relatives à la situation des restes des petits vertébrés par couches et par décapages. Le tableau 2 présente le décompte de ces vestiges par couches pour le sous-carré L6C, tandis que la figure 1 illustre l'évolution des concentrations de chaque taxon dans ce même sous-carré pour chaque décapage. Grâce à ces décapages, on constate que les petits mammifères se distribuent de façon hétérogène au sein de la couche 4 . En réalisant une analyse statistique basée sur une méthode de regroupement («cluster analysis») et le coefficient de dissimilarité de Bray-Curtis, on met en évidence une succession d'ensembles (notés de $B$ à $F$ ) qui diffère de l'archéostratigraphie. La couche 4 se caractérise

\section{Espèces décrites par Passemard (1936) \\ Espèces \\ identifiées}

\section{Groupe}

Insectivore

Talpa sp.

Sorex sp.

Sorex coronatus-araneus

Rongeur

Petit carnivore

Batracien
Apodemus sylvaticus

Arvicola amphibius

Arvicola terrestris

Arvicola sapidus

Castor fiber

Microtus agrestis

Microtus arvalis

Microtus agrestis/arvalis

Microtus Terricola duodecimcostatus

Microtus Terricola pyrenaicus

Microtus Terricola subterraneus

Microtus Terricola sp.

Mustela nivalis

$\mathrm{X}$

Mustela erminea

Rana sp.

Bufo sp.

$\mathrm{x}$
$\mathrm{X}$

1

3

64

3

30

11

2

2

5

1

2

1

$\mathrm{x}$

$\mathrm{x}$

\author{
Tableau 1 - Présence et \\ décomptes du nombre \\ minimal d'individus de petits \\ vertébrés provenant de la \\ couche inter FM-FS du site \\ d'Olha I (Pyrénées- \\ Atlantiques). \\ Table 1 - Presence of small \\ vertebrate from the level "inter \\ FM-FS" of Olha 1 (Pyrénées- \\ Atlantiques) identified by \\ Passemard (1936) as well as \\ the minimal number of \\ individuals for small vertebrate \\ identified during the \\ reevaluation.
}




\begin{tabular}{|c|c|c|c|c|c|c|c|c|c|}
\hline & $\begin{array}{l}\text { Microtus } \\
\text { indet. }\end{array}$ & $\begin{array}{c}\text { Microtus } \\
\text { arvalis/agrestis }\end{array}$ & $\begin{array}{c}\text { Microtus } \\
\text { oeconomus }\end{array}$ & $\begin{array}{c}\text { Arvicola } \text { cf. } \\
\text { terrestris }\end{array}$ & $\begin{array}{l}\text { Chionomys } \\
\text { nivalis }\end{array}$ & $\begin{array}{l}\text { Apodemus } \\
\text { sylvaticus }\end{array}$ & $\begin{array}{l}\text { Eliomys } \\
\text { quercinus }\end{array}$ & $\begin{array}{l}\text { Sicista } \\
\text { betulina }\end{array}$ & $\begin{array}{l}\text { Myodes } \\
\text { glareolus }\end{array}$ \\
\hline & $\mathrm{N}$ ml inf & $\mathrm{N} \mathrm{ml} \inf$ & $\mathrm{N} \mathrm{m1} \mathrm{inf}$ & $\mathrm{N} \mathrm{ml} \inf$ & $\mathrm{N} \mathrm{ml} \inf$ & $\begin{array}{l}\text { Mand } \\
+\max \\
\end{array}$ & $\begin{array}{l}\text { Mand } \\
+\max \\
\end{array}$ & $\begin{array}{l}\text { Mand } \\
+\max \end{array}$ & NR \\
\hline Couche 1 & 0 & 0 & 0 & 0 & 0 & 0 & 0 & 0 & 0 \\
\hline Couche 2 & 1 & 2 & 0 & 1 & 0 & 0 & 0 & 0 & 0 \\
\hline Couche 3 & 4 & 202 & 3 & 3 & 0 & 16 & 0 & 0 & 1 \\
\hline Couche 4 & 23 & 586 & 223 & 38 & 1 & 10 & 1 & 2 & 0 \\
\hline Couche 5 & 13 & 142 & 51 & 41 & 0 & 0 & 4 & 2 & 0 \\
\hline
\end{tabular}

\begin{tabular}{l|cccc} 
& $\begin{array}{c}\text { Sorex } \\
\text { coronatus- } \\
\text { araneus } \\
\text { mand }\end{array}$ & $\begin{array}{c}\text { Sorex } \\
\text { minutus }\end{array}$ & $\begin{array}{c}\text { Crocidura } \\
\text { indet. }\end{array}$ & $\begin{array}{c}\text { Neomys } \\
\text { Indet. }\end{array}$ \\
\hline mand & mand & mand \\
Couche 1 & 0 & 0 & 0 & 0 \\
Couche 2 & 0 & 0 & 0 & 0 \\
Couche 3 & 10 & 5 & 8 & 0 \\
Couche 5 & 64 & 22 & 0 & 9 \\
& 5 & 1 & 0 & 0
\end{tabular}

Tableau 2 - Décompte des restes de petits vertébrés provenant du site de Peyrazet (Lot) selon leurs appartenances aux couches archéologiques.

Table 2 - Remains of small vertebrate from Peyrazet (Lot) detailed according to archaeological levels.

par la présence de quatre ensembles dont deux (le B et le E) qui incorporent aussi des décapages provenant des couches 3 et 5 . L'ensemble $D$ de cette couche se démarque notamment par la présence de nombreux insectivores dont des musaraignes aquatiques (Neomys $\mathrm{sp}$.) et des musaraignes communes ou couronnées (Sorex coronatus-araneus), ainsi que de restes de sicistes (Sicista betulina) et de lérots (Eliomys quercinus). Cette association traduit un milieu marécageux, qui disparaît dans la partie supérieure de la couche 4 (ensemble $\mathrm{C}$ ). Par ailleurs, cette analyse par décapages donne la possibilité de réfléchir sur la réalité de certaines associations. II est par exemple notable dans le tableau 2 que la couche 4 associe le campagnol des neiges (Chionomys nivalis) avec une musaraigne aquatique (Neomys $\mathrm{sp}$.), taxons qui traduisent deux environnements différents. Néanmoins, l'analyse par décapage suggère que ces deux taxons ne se côtoient pas. Les décapages, dans cet exemple, permettent de proposer une biostratigraphie évitant l'effet palimpseste produit par la couche archéologique.

La séquence du gisement des Pradelles (Charente) (Maureille et al. 2010) est datée de la fin du stade isotopique 4 et du début du 3. Deux taxons sont majoritaires, le campagnol des hauteurs (Microtus gregalis) et le campagnol des champs (Microtus arvalis) ; ils représentent environ $85 \%$ des individus déterminés (Royer et al. 2013). Considérons uniquement le niveau 5 dont les restes de grands mammifères sont principalement accumulés par des grands carnivores (Costamagno et al. 2008). Ce niveau 5 est perçu comme homogène aussi bien d'un point de vue lithostratigraphique qu'archéologique. D'après les rongeurs, il est possible d'interpréter différemment ce niveau soit en tenant compte de son

intégralité soit en le divisant en deux sous-niveaux (tabl. 3). En considérant l'intégralité du niveau, on remarque que le nombre d'individus identifiés comme étant des campagnols des champs ( $M$. arvalis) augmente, tandis que la présence du campagnol des hauteurs ( $M$. gregalis) est toujours dominante, ce qui traduit un environnement continental steppique sec. Néanmoins, les proportions de ces rongeurs diffèrent si on subdivise ce niveau 5 en deux sous-niveaux (5-1 et 5-2). Dans le sous-niveau 5-1, la présence du campagnol des hauteurs domine face à celle du campagnol des champs, tandis que dans le sous-niveau 5-2, ce rapport est inversé. Ce second sous-niveau se différencie par ailleurs avec la présence du hamster migrateur (Cricetulus migratorius). Comparés à ceux du sous-niveau $5-1$, ces résultats conduisent à suggérer que le sous-niveau 5-2 a enregistré une amélioration climatique.

\section{5 - Apports des décapages et application sur le terrain}

L'utilisation systématique des décapages présente l'avantage de définir spatialement l'origine des micro-vestiges, de façon indépendante des couches archéologiques et géologiques, et offre la possibilité d'y réaffecter les objets ayant été enregistrés avec des coordonnées cartésiennes (fig. 2). Ce mode d'enregistrement permet ainsi :

1) d'établir une biostratigraphie fine et indépendante, qui n'est pas biaisée par les découpages litho- ou archéostratigraphique (fig. 2) et qui est corrélable avec ces dernières, comme cela a été réalisé dans de rares études de petits vertébrés (Cochard 2004 ; Cuenca-Bescós et al. 2011 ; Pasty et al. 2012); 


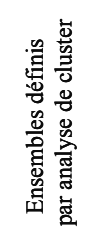

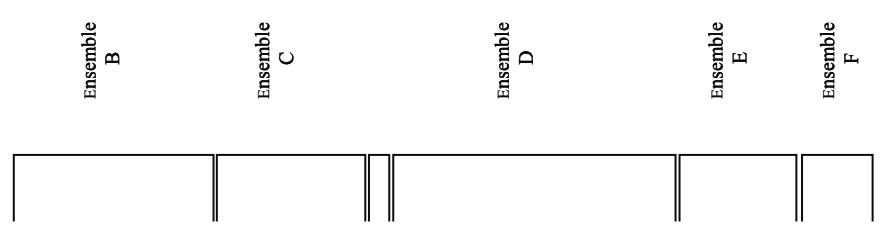

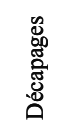

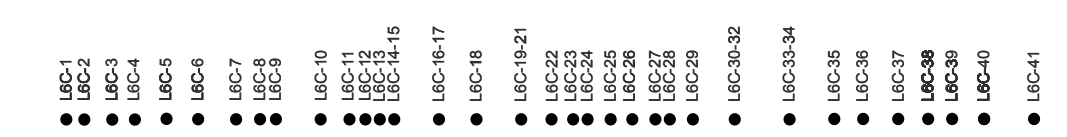

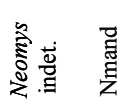
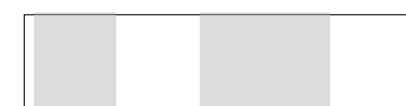

Hi

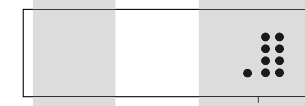

๘⿳亠幺𠃌

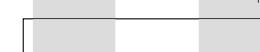

\section{i}

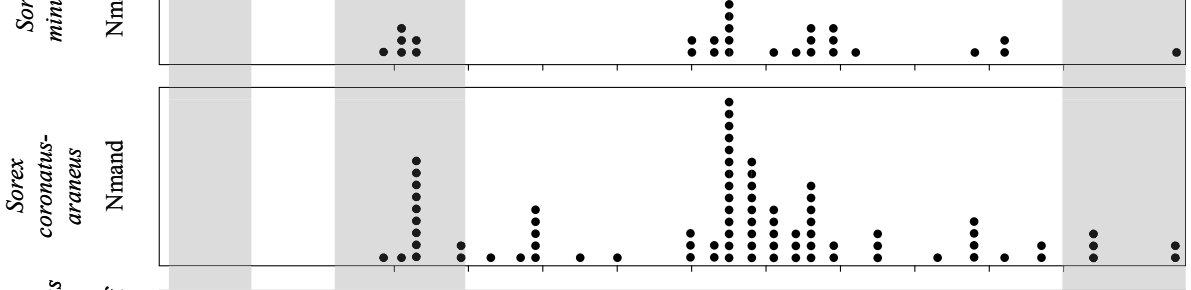

II

岁

II

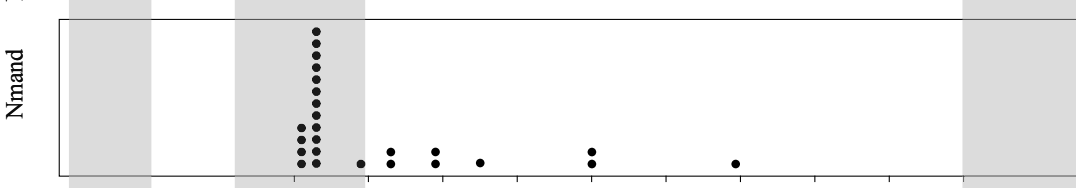

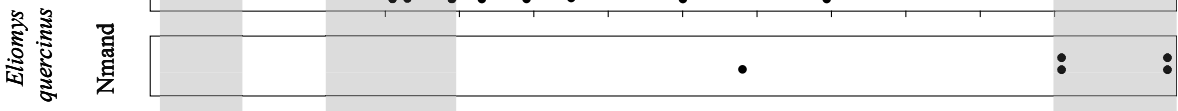
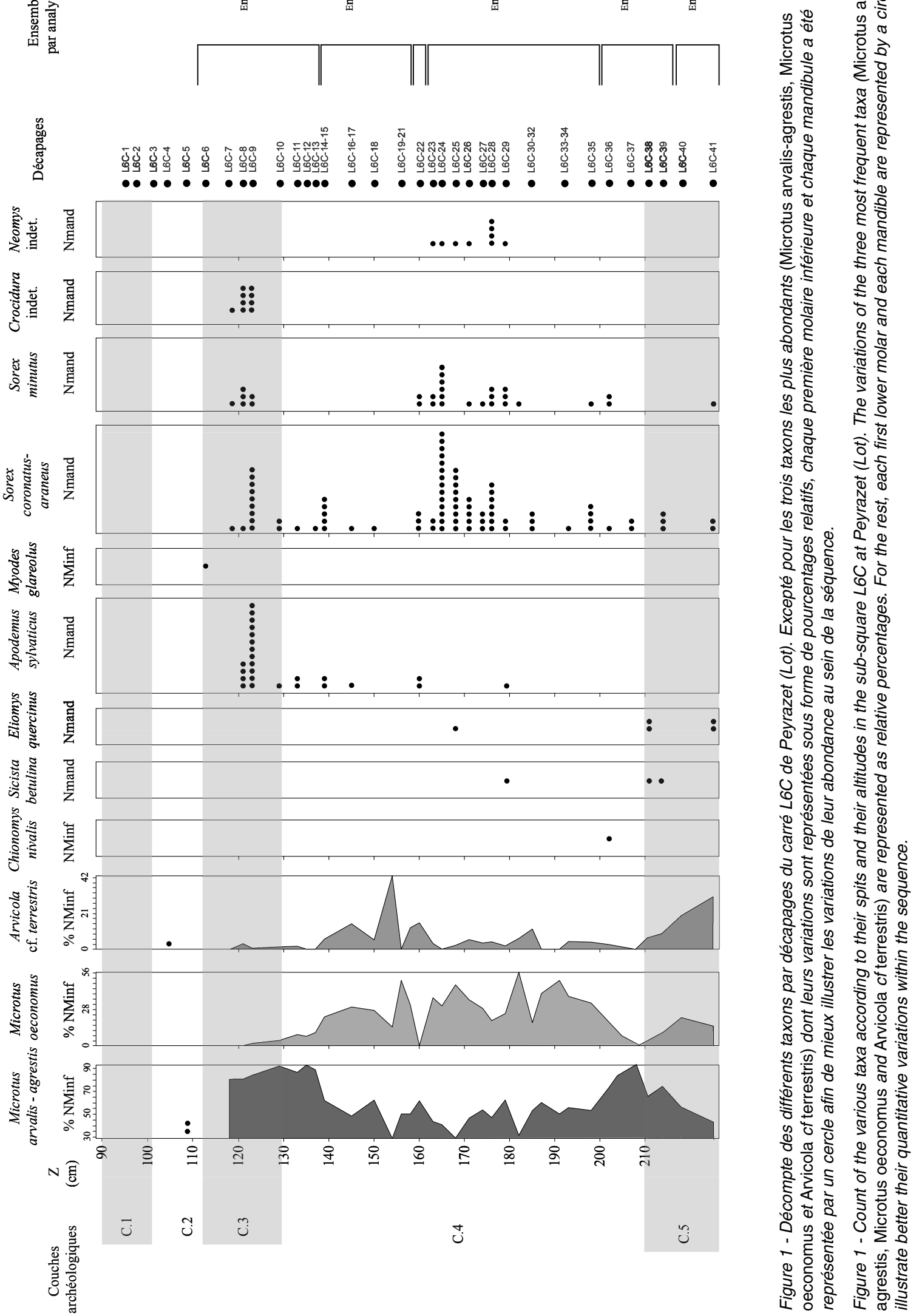


\begin{tabular}{c|cccccccc|c|c}
\cline { 2 - 9 } & Niveau & Niveau & Niveau & Niveau & Niveau & Niveau & Niveau & Niveau & Total \\
Espèces / Niveaux & $\mathbf{2 / 1}$ & $\mathbf{2 A}$ & $\mathbf{2 B}$ & $\mathbf{4 A}$ & $\mathbf{4 B}$ & $\mathbf{5 - 1}$ & $\mathbf{5 - 2}$ & $\mathbf{5}$ total & (NMI=1250) \\
\hline Apodemus sylvaticus & 0 & 0 & 0 & 1 & 1 & 0 & 1 & 1 & $\mathbf{3}$ \\
Arvicola terrestris & 1 & 4 & 1 & 1 & 2 & 2 & 5 & 7 & $\mathbf{1 7}$ \\
Cricetelus migratorius & 0 & 0 & 0 & 1 & 0 & 0 & 4 & 4 & $\mathbf{5}$ \\
Dicrostonyx torquatus & 0 & 1 & 1 & 4 & 64 & 26 & 18 & 44 & $\mathbf{1 4 2}$ \\
Eliomys quercinus & 0 & 0 & 0 & 0 & 0 & 1 & 0 & 1 & $\mathbf{1}$ \\
Glis glis & 0 & 0 & 0 & 0 & 1 & 0 & 0 & 0 & $\mathbf{1}$ \\
Marmota marmota & 0 & 0 & 0 & 1 & 2 & 0 & 0 & 0 & $\mathbf{4}$ \\
Microtus arvalis & 4 & 13 & 3 & 14 & 39 & 31 & 147 & 178 & $\mathbf{2 7 2}$ \\
Microtus & & & & & & & & & \\
arvalis/agrestis & 0 & 0 & 0 & 0 & 3 & 1 & 3 & 4 & $\mathbf{8}$ \\
Microtus gregalis & 7 & 35 & 8 & 26 & 296 & 168 & 120 & 288 & $\mathbf{7 8 2}$ \\
Microtus oeconomus & 0 & 0 & 1 & 2 & 3 & 1 & 7 & 8 & $\mathbf{1 5}$
\end{tabular}

Tableau 3 - Décompte du nombre minimal d'individus pour les rongeurs du site des Pradelles. Le niveau « 5 total » correspond à l'addition des deux sous-niveaux 5-1 et 5-2.

Table 3 - Minimal number of individuals for rodents at Les Pradelles (Charente). The level "5 total" corresponds to both sub-levels 5-1 and 5-2.

2) de développer une démarche confrontant les répartitions des objets non-coordonnés (restes de petits vertébrés mais également tous les autres microvestiges tels que les vestiges lithiques et les os brûlés) ainsi que celles des pièces coordonnées. Un exemple a été obtenu à partir de l'étude du gisement Tardiglaciaire de Peyrazet (Lot), pour lequel une absence de corrélation entre les quantités de matériels lithiques (accumulé par l'Homme) et celles de petits mammifères (accumulé par un prédateur nonhumain) a été mise en évidence (Rho $=0,10 ; p=0,55)$. En revanche, il existe une absence de corrélation entre les quantités de matériel lithique et celles de poissons $(\mathrm{Rho}=$ 0,$10 ; p=0,52$ ) et une corrélation significative entre les quantités de petits mammifères et celles de poissons (Rho $=0,57 ; p<0,0001)$. Ces résultats suggèrent donc qu'une grande partie de ces derniers ont, eux aussi, été accumulés par des prédateurs non-humain. À partir de $-150 \mathrm{~cm}$, les restes de petits vertébrés sont toujours présents en quantité importante, tandis que la densité des pièces lithiques est faible, ce qui témoigne d'une occupation de la grotte principalement par des prédateurs non-humains (fig. 3). Cette méthode offre donc la possibilité de caractériser les phases d'occupations, mais également d'inoccupations, d'un site par l'Homme grâce à l'analyse de niveaux qui sont considérés comme archéologiquement stérile.

Si cette démarche est réalisée sur l'ensemble du site fouillé, elle peut aussi permettre de réaliser une étude spatiale des restes de petits vertébrés, qui peut être comparée avec celles d'autres vestiges. Par exemple, la couche Illa magdalénienne du Taillis-des-Coteaux (Vienne) (Primault et al. 2010) présente une répartition similaire des restes de rongeurs, d'oiseaux et de poissons, localisés au niveau du porche de l'entrée de grotte. Cette distribution diffère de celle des vestiges liés aux activités anthropiques qui sont principalement répartis à l'intérieur de la grotte et sur le talus (Rambaud et al. 2011). Cette répartition différentielle a permis de suggérer que les vestiges de poissons et d'oiseaux, comme ceux des rongeurs, résultent principalement d'une accumulation liée à l'activité d'un prédateur non-humain ;

3) de produire des informations neutres permettant une réévaluation ultérieure de la collection et pouvant aussi être confrontées à une révision des autres vestiges archéologiques.

Aujourd'hui, le tamisage systématique est souvent critiqué car c'est une opération fastidieuse et consommatrice en temps. Néanmoins, cette opération est nécessaire pour récupérer des vestiges archéologiques qui ont échappé à la fouille, tels que certains restes lithiques ou osseux, indispensables pour la compréhension des comportements humains (e.g. Freeman et al. 1998 ; Soressi et Tavormina 2011 ; Val et Mallye 2011). Il est important de souligner que le mode d'enregistrement par décapages est certes légèrement plus long qu'un traitement par couche, mais il permet un gain important d'informations. La récupération des restes de petits vertébrés sur le terrain dépend non seulement des méthodes de fouilles, mais aussi des mailles de tamis utilisées par les préhistoriens. Ces derniers utilisent des mailles entre 3 et $1,5 \mathrm{~mm}$ pour récupérer les plus petites armatures lithiques, tandis que les spécialistes des petits vertébrés recommandent des mailles comprises entre 1,2 et $0,5 \mathrm{~mm}$ selon la fragmentation des vestiges. L'idéal est bien évidemment de tamiser systématiquement à $1,2 \mathrm{~mm}$ ou moins, comme cela est déjà pratiqué sur d'autres gisements comme à Atapuerca (maille de 0,5 mm - com. pers. G. CuencaBescós). Dans le cas où cette démarche ne peut être appliquée, deux autres stratégies, qui dépendent du site et des objectifs de la fouille, peuvent être développées. Dans 


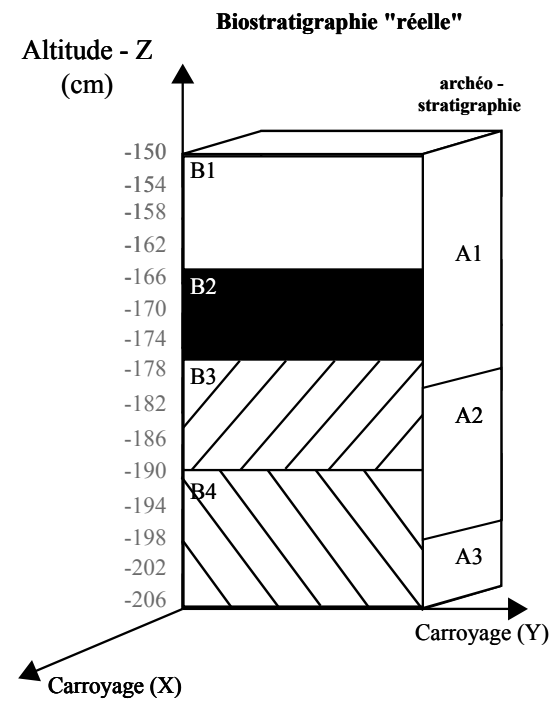

\section{Biostratigraphie obtenue par l'archéostratigraphie}

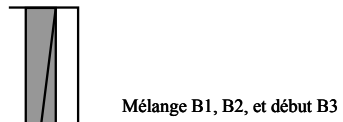

Mélange B3 et B4

Reflète B4

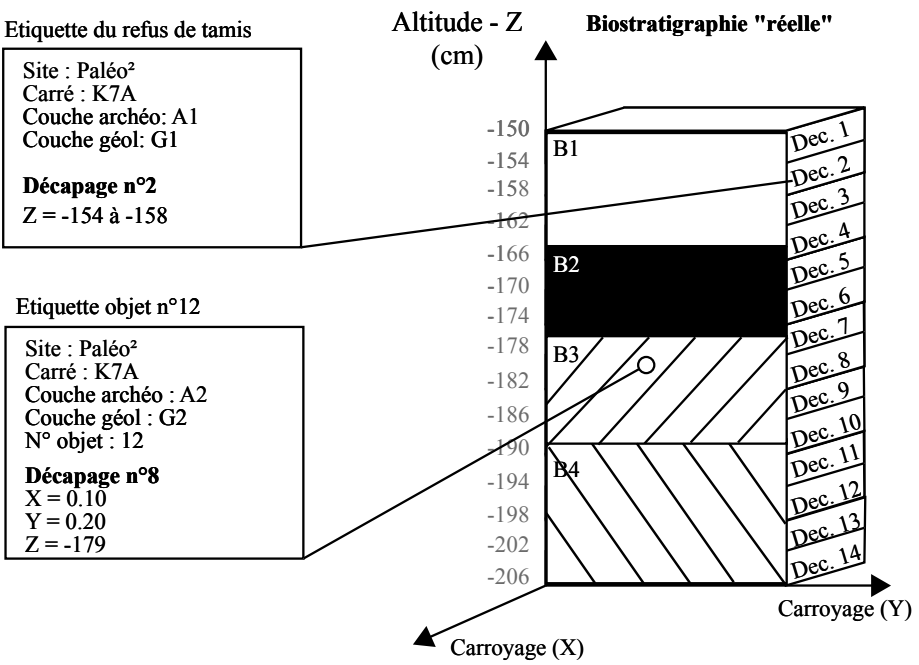

\section{Biostratigraphie obtenue par les décapages}

Figure 2 - Cas théorique permettant de comparer une biostratigraphie obtenue à partir des micromammifères recueillis dans les différentes archéostrates du carré K7A (schéma A) et une biostratigraphie construite à partir de récoltes effectuées dans le même carré K7A mais selon des décapages (Dec) successifs indépendants de l'archéostratigraphie (schéma $B$ ).

Figure 2 - Theoretical illustration presenting the difference between a biostratigraphy constructed by using archaeostratigraphical levels from the square K7A (Schema $A$ ) and a biostratigraphy obtained by using successive splits (Dec) from the square K7A, which are independent from the archaeostratigraphy (Schema B).

le cadre d'une fouille favorisant l'analyse spatiale, il convient de s'orienter vers un tamisage systématique, qui peut-être réalisé avec une maille grossière entre 1,2 et 1,8 $\mathrm{mm}$, ce qui permet d'appréhender la répartition des densités de vestiges. Cette démarche devra être complétée par des colonnes de prélèvements qui seront tamisées avec une maille plus petite afin d'affiner les reconstitutions environnementales grâce aux restes des plus petites espèces. Dans le cadre d'une fouille n'impliquant pas une démarche spatiale, telle que la reprise d'une coupe, il est possible de réaliser des colonnes de prélèvements tamisées avec une maille de tamis fine, colonnes qui seront régulièrement réparties le long de la coupe (par exemple un sous-carré de fouille tous les mètres). Ces deux stratégies présentent l'avantage de pouvoir s'appuyer sur un enregistrement par décapages qui s'insère dans le 


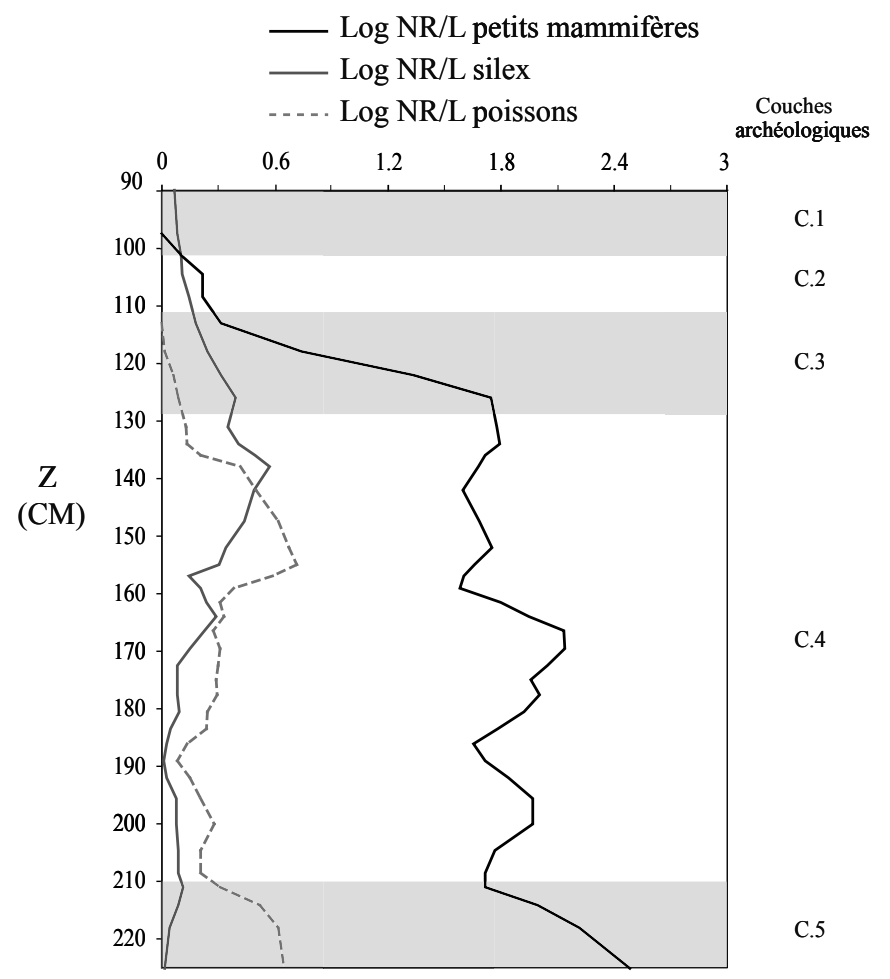

Figure 3 - Logarithme (Log) des variations des quantités de restes de petits mammifères, de lithiques et de poissons par litre de sédiments obtenus par décapages du carré L6C de Peyrazet (Lot) selon leurs profondeurs. NR : Nombre de restes ; $L$ : Litre de sédiments ; $Z$ : Altitude en centimètre. Les alternances grisées et non-grisées illustrent les couches archéologiques.

Figure 3 - Variations of densities of the rodents, fish and lithic remains by liter of sediment (expressed in logarithm) obtained by spits from the sub-square L6C of Peyrazet (Lot) according to their altitudes. NR: Number of remains; L: Liter of sediments; Z: altitude in centimeters. The greyed and non-greyed alternations illustrate the archaeological levels

carroyage du site permettant d'acquérir des données adéquates pour la microfaune et de les corréler directement avec les données archéologiques et géologiques.

\section{6 - Conclusion}

Les restes de petits vertébrés sont porteurs de nombreuses informations, aussi bien environnementales et archéozoologiques que taphonomiques. Cependant, leur potentiel informatif est dépendant des méthodes de fouilles utilisées pour les recueillir. Du fait de leur petite taille, les restes de petits vertébrés sont très rarement enregistrés avec un système de coordonnées cartésiennes au sein des gisements archéologiques, car ils sont très majoritairement collectés lors du tri des refus de tamis. Cet article souligne la nécessité d'acquérir des informations spatiales neutres pour ce matériel via notamment l'utilisation de décapages. Chacun de ces derniers représente un ensemble de restes provenant du volume d'un hexaèdre référencé spatialement au sein du gisement. Ils sont indépendants des couches archéologiques et géologiques, ce qui permet d'étudier la répartition spatiale des restes de petits vertébrés, de proposer des biostratigraphies basées sur ces vestiges et d'enregistrer les informations nécessaires pour permettre une confrontation avec les autres vestiges archéologiques et une éventuelle réévaluation ultérieure de la collection.

\section{Remerciements}

Je remercie Odile Romain de m'avoir permis d'accéder à la collection d'Olha 1 (Pyrénées-Atlantiques) au Musée de l'Homme, Bruno Maureille et Alan Mann pour la collection des Pradelles (Charente), ainsi que Mathieu Langlais pour celle de Peyrazet (Lot). Je remercie aussi Véronique Laroulandie, Luca Sitzia, Emmanuelle Stoetzel et JeanPierre Texier pour leurs précieux commentaires qui ont permis d'améliorer ce texte.

\section{Références bibliographiques}

ANDREWS P. 1990 - Owls, caves, and fossils: predation, preservation, and accumulation of small mammal bones in caves, with an analysis of the Pleistocene cave faunas from Westbury-sub-Mendip, Somerset, UK. University of Chicago Press, $231 \mathrm{p}$.

AUFFRAY J.C., RENAUD S. et CLAUDE J. 2009 - Rodent biodiversity in changing environments. Kasetsart Journal, Natural Sciences, 43, 83-93.

BORDES F. et BOURGON M. 1951 - Le gisement du Pech de l'Azé Nord. Campagnes 1950-1951. Les couches inférieures à Rhinoceros Mercki. Bulletin de la Société préhistorique de France, p. 520-538.

BORDES F., RIGAUD J.-P. et SONNEVILLE-BORDES (de) D. 1972 - Des buts, problèmes et limites de l'archéologie paléolithique. Quaternaria, 16, p. 15-34.

BOUCHUD J. 1952 - Étude des rongeurs et des oiseaux de l'abri Castanet. Bulletin de la Société préhistorique de France, 49, p. 267-271.

CHALINE J. 1972 - Les rongeurs du Pléistocène moyen et supérieur de France. Cahiers de Paléontologie. CNRS, Paris, $400 \mathrm{p}$.

CHALINE J. 1977 - Les rongeurs au pilori ? Analyse critique des reconstitutions paléoécologiques en microthériodontologie. In : LAVILLE $\mathrm{H}$. et RENAULT-MISKOVSKY J. (eds.), Approche écologique de l'Homme fossile. Paris, p. 77-81.

COCHARD D. 2004 - Étude préliminaire des micromammifères des niveaux Paléolithique moyen de Chez Pinaud à Jonzac (Charente, France). In: AIRVAUX J. (ed.) Le site Paléolithique de Chez-Pinaud à Jonzac, Charente-Maritime. Préhistoire du Sud-Ouest, p. 157-162. 
COSTAMAGNO S., BEAUVAL C., LANGE-BADRÉ B., VANDERMEERSCH B., MANN A. et MAUREILLE B. 2008 - Homme ou carnivores ? Protocole d'étude d'ensembles osseux mixtes : l'exemple du gisement moustérien des Pradelles (Marillac-le-Franc, Charente). P@lethnologie, 1, p. 372-400.

CUENCA-BESCÓS G., STRAUS L.G., GONZÁLEZ MORALES M.R. et GARCÍA PIMIENTA J.C. 2009 - The reconstruction of past environments through small mammals: from the Mousterian to the Bronze Age in El Mirón Cave (Cantabria, Spain). Journal of Archaeological Science, 36, 4, p. 947-955.

CUENCA-BESCÓS G., MELERO-RUBIO M., ROFES J., MARTÍNEZ I., ARSUAGA J.L., BLAIN H.A., LÓPEZGARCÍA J.M., CARBONELL E. et BERMUDEZ DE CASTRO J.M. 2011 - The Early-Middle Pleistocene environmental and climatic change and the human expansion in Western Europe: A case study with small vertebrates (Gran Dolina, Atapuerca, Spain). Journal of Human Evolution, 60, 4, p. 481-491.

FREEMAN L.G., GONZALEZ ECHEGARAY J., POKINES J.T., STETTLER H.K. et KRUPA M. 1998 - Tamisage ultra fin et récupération de l'outillage : observations réalisées à El Juyo (Espagne cantabrique). L'Anthropologie, 102, 1, p. 35-44.

HARLÉ E. 1906 - Lemming à collier (Myodes torquatus $P$.) de Teyjat (Dordogne). Bulletin de la Société Géologique de France, 6, p. 11.

HEDBERG H.D. 1979 - Guide stratigraphique international. Classification, terminologie et règles de procédure. Paris, $233 \mathrm{p}$.

HERNÁNDEZ FERNÁNDEZ M. 2006 - Rodent paleofaunas as indicators of climatic change in Europe during the last 125,000 years. Quaternary Research, 65, 2, p. 308-323.

JEANNET M. 1978 - Recherches sur les rongeurs quaternaires en 1978. Nouvelles Archives du Museum d'Histoire naturelle de Lyon, 16, suppl., p. 71-74.

JEANNET M. 2010 - L'écologie quantifiée. Essai de description de l'environnement continental à l'aide des microvertébrés. Préhistoires Méditerranéennes, 1, p. 11-34.

LANGLAIS M., LAROULANDIE V., AVEC LA COLLABORATION DE, BRUXELLES L., CHALARD P., COCHARD D., COSTAMAGNO S., DELFOUR G., KUNTZ D., LE GALL O., PÉTILLON J.-M. et QUEFFELEC A. 2009 Les fouilles de la grotte-abri de Peyrazet (Creysse, Lot) : nouvelles données pour le Tardiglaciaire quercinois. Bulletin de la Société préhistorique française, 106, 1, p. 150-152.

LAPLACE-JAURETCHE G. 1971 - De l'application des coordonnées cartésiennes à la fouille stratigraphique. Munibe, 23, 2-3, p. 223-236.
LAPLACE-JAURETCHE G. et MÉROC L. 1954 - Application des coordonnées cartésiennes à la fouille d'un gisement. Bulletin de la Société préhistorique de France, 51, 1-2, p. 58-66.

LEROI-GOURHAN A. 1950 - La grotte du Loup, Arcy-surCure (Yonne). Bulletin de la Société préhistorique de France, 47, 5, p. 268-280.

LEROI-GOURHAN A. 1988 - Dictionnaire de la Préhistoire. Presses universitaires de France.

LE LOUARN H. et QUÉRÉ J.P. 2003 - Les rongeurs de France : faunistique et biologie. Editions Quae, $260 \mathrm{p}$.

LÓPEZ-GARCÍA J.M., BLAIN H.A., CUENCA-BESCÓS G., RUIZ-ZAPATA M.B., DORADO-VALIÑO M., GIL-GARCÍA M.J., VALDEOLMILLOS A., ORTEGA A.I., CARRETERO J.M., ARSUAGA J.L., BERMÚDEZ DE CASTRO J.M. et CARBONELL E. 2010 - Palaeoenvironmental and palaeoclimatic reconstruction of the latest Pleistocene of $\mathrm{El}$ Portalón site, Sierra de Atapuerca, northwestern Spain. Palaeogeography, Palaeoclimatology, Palaeoecology, 292, 3, p. 453-464.

LUMLEY (de) H. 1969 - Les Méthodes de fouilles préhistoriques. Mémoires de la Société Préhistorique Française, 7, p. 13-15.

LUMLEY (de) H. 1972 - Méthodes de fouilles et d'études d'un gisement préhistorique. Etudes Quaternaires, 1, p. 7-17.

MARQUET J.-C. 1993 - Paléoenvironnement et chronologie des sites du domaine Atlantique français d'âge Pléistocène moyen et supérieur d'après l'étude des rongeurs. Tours: Les Cahiers de la Claise, Supplément 2, $330 \mathrm{p}$.

MAUREILLE B., MANN A., BEAUVAL C., BORDES J.G., BOURGUIGNON L., COSTAMAGNO S., COUCHOUD I., FAUQUIGNON J., GARRALDA BENAJES M.D., GEIGL E.M., GRÜN R., GUIBERT P., LACRAMPE F., LAROULANDIE V., MARQUET J.C., MEIGNEN L., MUSSINI C., RENDU W., ROYER A., SEGUIN G. et TEXIER J.-P. 2010 - Les Pradelles à Marillac-le-Franc (Charente). Fouilles 2001-2007 : nouveaux résultats et synthèse. In: BUISSON-CATIL J. et PRIMAULT J. (eds.), Préhistoire entre Vienne et Charente. Hommes et sociétés du Paléolithique. Chauvigny: Association des Publications Chauvinoises, p. 145-162.

MAYHEW D.F. 1977 - Avian predators as accumulators of fossil mammal material. Boreas, 6, p. 25-31.

PASSEMARD E. 1936 - Le moustérien à l'Abri Olha en pays basque. Société d'Impressions Typographiques. $46 \mathrm{p}$.

PASTY J.-F., COSTAMAGNO S., MISTROT V., LAROULANDIE V., ALIX P., BALLUT C., PASTY-VANDE WALLE H. et MURAT R. 2012 - Implications biostratigraphiques et paléoenvironnementales des occupations du Paléolithique moyen et du Châtelperronien du site de La Tour Fondue à Chauriat (Puy-de-Dôme, France). Paleo, 23, p. 189-222. 
PRIMAULT J., BERTHET A.-L., BROU L., DELFOUR G., GABILLEAU J., GRIGGO C., GUÉRIN S., GAMBIER D., HOUMARD C., JEANNET M., LACRAMPE F., LANGLAIS M., LAROULANDIE V., LIARD M., LIOLIOS D., LOMPRÉ A., LUCQUIN A., MISTROT V., RAMBAUD D., SCHMITT A., SOLER L., TABORIN Y. et VISSAC C. 2010 - La grotte du Taillis-des-Coteaux à Antigny (Vienne). In : BUISSONCATIL J. et PRIMAULT J. (eds.), Préhistoire entre Vienne et Charente. Hommes et sociétés du Paléolithique. Chauvigny: Association des Publications Chauvinoises, p. 271-293.

RAMBAUD D., LAROULANDIE V., PRIMAULT J. et BEAREZ P. 2011 - Les poissons et les oiseaux du Taillis des Coteaux (Antigny, Vienne), niveaux magdaléniens : origine naturelle ou culturelle ? In : LAROULANDIE V., MALLYE J.-B. et DENYS C. (eds.), Taphonomie des petits vertébrés: référentiels et transferts aux fossiles. Actes de la Table Ronde du RTP Taphonomie, Talence 20-21 october 2009. Oxford: BAR International Series, p. 167-179.

REINECK H.-E. et SINGH I.B. 1975 - Depositional sedimentary environments: with reference to Terrigenous clastics. Berlin: Springer-Verlag, $439 \mathrm{p}$.

ROYER A., LÉCUYER C., MONTUIRE S., ESCARGUEL G., FOUREL F., MANN A. et MAUREILLE B. 2013 - Late Pleistocene (MIS 3-4) climate inferred from micromammal communities and $\delta^{18} \mathrm{O}$ of rodents from Les Pradelles, France. Quaternary Research, 80, p. 113-124.
SIMARD S. 1966 - Etude paléontologique des micromammifères de la grotte du Regourdou (Montignac, Dordogne). Faculté des Sciences de l'Université de Paris, 1966. $118 \mathrm{p}$.

SONNEVILLE-BORDES (de) D. et MORTUREUX B. 1955 L'abri Caminade, commune de La Canéda (Dordogne). Bulletin de la Societé préhistorique de France, 52, p. 609-619.

SORESSI M. et TAVORMINA C. 2011 - L'intérêt du tri du refus de tamis de maille $5 \mathrm{~mm}$ et $2 \mathrm{~mm}$ dans l'analyse d'assemblages lithiques du Protoaurignacien et de l'Aurignacien ancien. Bulletin de liaison et d'information Association des archéologues ; Direction des antiquités Poitou-Charentes 40, p. 9-16.

STAHL P.W. 1996 - The recovery and interpretation of microvertebrate bone assemblages from archaeological contexts. Journal of Archaeological Method and Theory, 3 , 1, p. 31-75.

VAL A. et MALLYE J.-B. 2011 - Taphonomie du fouilleur : influence de la maille de tamis sur la représentation anatomique des petits animaux à fourrure. In : DENYS C.,

LAROULANDIE V. et MALLYE J.-B. (eds.), Taphonomie des Petits Vertébrés: Référentiels et Transferts aux Fossiles. BAR International Series, p. 93-100.

VAUFREY R. 1956 - Fouilles et « Archéologie sur le terrain ». Bulletin de la Société préhistorique de France, p. 491-503.

VILLA P. 2004 - Taphonomy and stratigraphy in European prehistory. Before farming, 1, p. 1-20. 